\title{
Theory of extraordinary transmission of light through quasiperiodic arrays of subwavelength holes
}

\author{
J. Bravo-Abad, ${ }^{1}$ A.I. Fernández-Domínguez, ${ }^{1}$ F.J. García-Vidal, ${ }^{1}$ and L. Martín-Moreno ${ }^{2}$ \\ ${ }^{1}$ Departamento de Física Teórica de la Materia Condensada, Universidad Autónoma de Madrid, E-28049 Madrid, Spain \\ ${ }^{2}$ Departamento de Física de la Materia Condensada, ICMA-CSIC, Universidad de Zaragoza, E-50009 Zaragoza, Spain
}

(Dated: May 7, 2019)

\begin{abstract}
By using a theoretical formalism able to work in both real and k-spaces, the physical origin of the phenomenon of extraordinary transmission of light through quasi-periodic arrays of holes is revealed. Long-range order present in a quasiperiodic array selects the wavevector(s) of the surface electromagnetic mode(s) that allows an efficient transmission of light through subwavelength holes.
\end{abstract}

PACS numbers: 42.79.Ag, 41.20.Jb, 42.25.Bs, 73.20.Mf

The phenomenon of extraordinary optical transmission (EOT) through periodic two-dimensional (2D) arrays of subwavelength holes milled in a metallic film [1] has sparked a great deal of interest due to both its fundamental implications and its broad range of potential applications. Subsequent experimental and theoretical works have concentrated on analyzing periodic structures $[2,3,44,[5,6,6,8,8,9$, 10]. However, very recently, several experimental studies showing EOT in quasiperiodic arrays of holes have been reported [11, 12, 13, 14]. These results suggest that the presence of long-range order in a $2 \mathrm{D}$ hole array is the key ingredient to observe EOT.

In this Letter we present a complete physical explanation of the EOT properties found in quasiperiodic distributions of subwavelength holes. This analysis is based on the comparison between the transmission properties of finite Penrose lattices of holes with those associated with periodic arrays. The picture that emerges from our theoretical study is that the physical origin of EOT is common for both periodic and quasiperiodic arrays. It relies on the excitation of surface electromagnetic (EM) modes decorating the metallic interfaces.

In this paper, our study is focused on analyzing the transmission properties of Penrose lattices exhibiting tenfold rotational symmetry, as those studied experimentally in Ref. 14]. As in the experimental structure, the hole radius is chosen to be $a=0.2 \mathrm{~mm}$, the thickness of the metallic film is $h=0.075 \mathrm{~mm}$ and the length of the rhombus side defining the Penrose tiling, $d$, is $d=1 \mathrm{~mm}$. In our simulations, metal is treated as a perfect conductor (i.e. with dielectric constant $\epsilon=-\infty$ ), which is an excellent approximation in the $\mathrm{THz}$ regime. In order to calculate the scattering properties and EM field distributions, we use a formalism based on a modal expansion of the fields at the hole openings [7], which allows treating efficiently large numbers of indentations, arbitrarily placed in a metal film.

Figure 1 shows the three different types of hole arrangements considered in this work. Left, center and right panels correspond to a periodic square lattice, a ten-fold Penrose lattice and a random distribution of cir- (a)

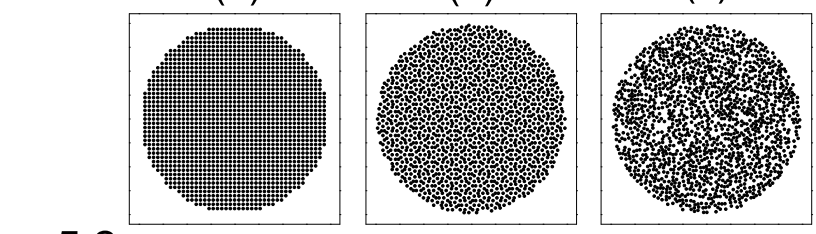

(b)

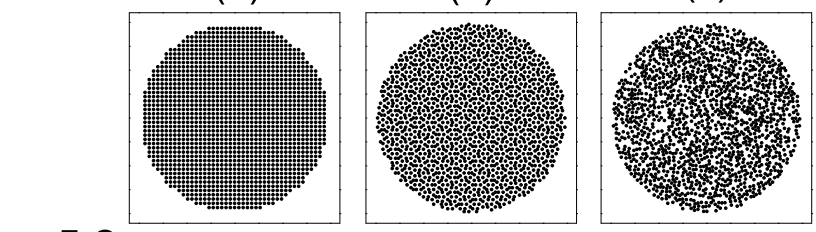

(c)

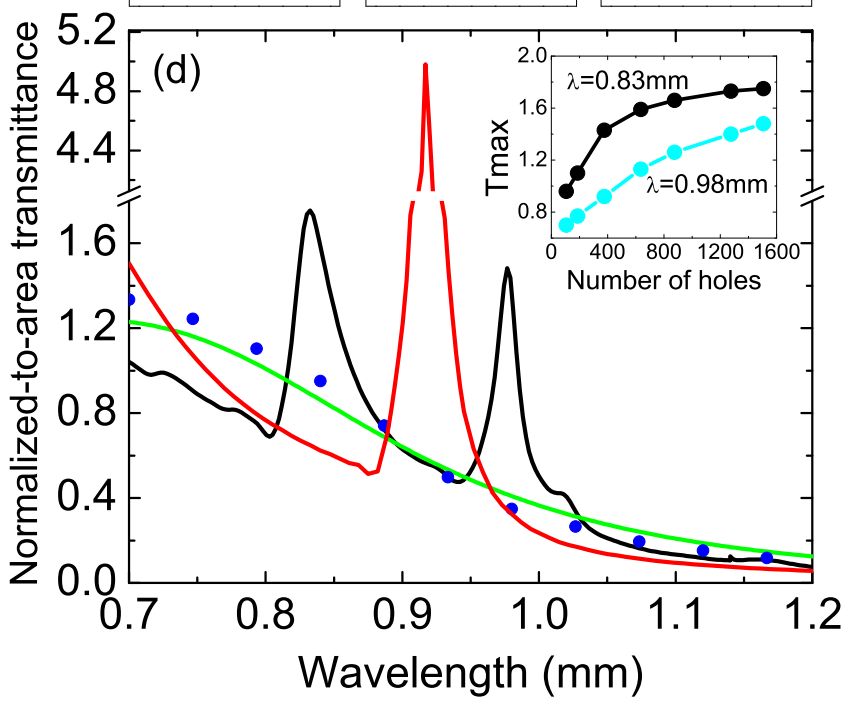

FIG. 1: (color-on-line) (a-c) Structures considered in this work. Square (left), Penrose (center) and random lattices (right). (d) Normalized-to-area transmittance $(T)$ spectra for: single hole (green line), square array (red line), Penrose lattice (black line) and a random configuration (blue dots). In all four cases, $a=0.2 \mathrm{~mm}, h=0.075 \mathrm{~mm}$ and $N=1506$. Inset in panel (d) shows the dependence with $N$ for $T$ at resonant peaks for the quasiperiodic array, $\lambda=0.83 \mathrm{~mm}$ (black dots) and $\lambda=0.98 \mathrm{~mm}$ (cyan dots).

cular holes, respectively. In all three cases, the number of holes $(N=1506)$, their diameter, the film thickness and the external radius of the circular array are the same. The coordinates in the Penrose lattice were generated by the Dual Generalized Method [15, 16]. The periodic structure is a circular portion of a square lattice with lattice parameter $P=0.89 \mathrm{~mm}$. In the disordered case, holes are randomly distributed but without allowing any 
interhole distance to be smaller than the minimum one found in the quasiperiodic case.

Figure 1d depicts the normal incidence transmission spectra computed for the three structures, along with the transmittance associated with a single hole (green line). In all cases, the transmittance is normalized to the flux of light impinging on the area occupied by the holes. In the spectral range considered, the single hole transmittance is a smooth decreasing function of the wavelength. In the ordered case (red line), the transmittance spectrum is also smooth, except close to the resonant peak appearing at $\lambda=0.92 \mathrm{~mm}$, where the normalizedto-area transmittance $(T)$ is about 5 for the geometrical parameters we are considering. This is the canonical EOT peak, appearing at a resonant wavelength slightly larger that the lattice parameter. As in the experiments, resonant transmission also appears when holes are arranged in a Penrose lattice (black curve in Fig. 1). In this case, maximum transmission values of about 1.5 are obtained at two resonant wavelengths, $\lambda=0.83 \mathrm{~mm}$ and $\lambda=0.98 \mathrm{~mm}$. The agreement between theory and experiment is remarkable (see Fig. 2c in Ref. 14]). On the other hand, blue dots in Fig. 1d demonstrate that EOT does not appear for any distribution of holes: the transmission spectrum for the random array does not show any resonant feature. This is just a representative example of disordered arrays; we have generated several random configurations finding always a non-resonant behavior.

The appearance of EOT can be related to the lattice structure in reciprocal space by extending arguments borrowed from the ordered case to different lattices, as follows. Following Ref. [7], EM fields in all space can be expressed in terms of the modal amplitudes of the waveguide modes right at the opening and the exit of the different holes $\left(E_{\alpha}(\mathbf{R})\right.$ and $E_{\alpha}^{\prime}(\mathbf{R})$, respectively, with $\mathbf{R}$ referring to the $2 \mathrm{D}$ array locations and $\alpha$ running over the modes inside the holes). These quantities can, in turn, be obtained by solving a coupled system of equations. In order to find the link between EOT and the structure factor of a given set of holes, $S(\mathbf{q})=\sum_{\mathbf{R}} \exp (-i \mathbf{q R})$, it is convenient to work with the Fourier components $E_{\alpha}(\mathbf{q})=\sum_{\mathbf{R}} \exp (-\imath \mathbf{q} \mathbf{R}) E_{\alpha}(\mathbf{R})$, which satisfy,

$$
\begin{aligned}
& \left(\Sigma_{n}(\mathbf{q})-\epsilon_{n}\right) E_{n}(\mathbf{q})-G_{n}^{V} E_{n}^{\prime}(\mathbf{q})=I_{n} S\left(\mathbf{q}-\mathbf{k}_{0}\right) \\
& \left(\Sigma_{n}^{\prime}(\mathbf{q})-\epsilon_{n}\right) E_{n}^{\prime}(\mathbf{q})-G_{n}^{V} E_{n}(\mathbf{q})=0
\end{aligned}
$$

where:

$$
\Sigma_{n}^{(\prime)}(\mathbf{q})=\frac{1}{E_{n}^{(\prime)}(\mathbf{q})} \sum_{m} \int d \mathbf{k} G_{m n ; \mathbf{k}} S(\mathbf{q}-\mathbf{k}) E_{m}^{(\prime)}(\mathbf{k})
$$

The expression for the different quantities can be straightforwardly obtained from the ones given in Ref. [7]. External illumination originates $I_{n} S\left(\mathbf{q}-\mathbf{k}_{0}\right), \mathbf{k}_{0}$ being the in-plane component of the incident wavevector. The term $\epsilon_{n}=-i Y_{n}\left(1+\Phi_{n}\right) /\left(1-\Phi_{n}\right)$, with $\Phi_{n} \equiv \exp \left(2 \imath q_{n} h\right)\left(Y_{n}\right.$ and $q_{n}$ being the admittance and the propagation constant of mode $n$ inside the holes, respectively), is related to the bouncing back and forth of EM fields inside the hole. $G_{n}^{V}=-2 i Y_{n} \sqrt{\Phi_{n}} /\left(1-\Phi_{n}\right)$ couples the input and exit sides of the hole. These magnitudes $\left(\epsilon_{n}\right.$ and $\left.G_{n}^{V}\right)$ show no dependence on parallel momentum, $\mathbf{k}$, as they do not couple modes in different holes and they are real quantities for subwavelength holes. The terms $\Sigma_{n}^{(\prime)}(\mathbf{q}) E_{n}^{(\prime)}(\mathbf{q})$ represent the scattering process that couples $E_{n}^{(\prime)}(\mathbf{q})$ to the continuum $E_{m}^{(\prime)}(\mathbf{k})$, the momentum difference being provided by the lattice through $S(\mathbf{q}-\mathbf{k})$. The amplitude of the process depends on $G_{m n ; \mathbf{k}}$ :

$$
G_{m n ; \mathbf{k}}=\frac{i}{(2 \pi)^{2}} \sum_{\sigma} Y_{\mathbf{k} \sigma}<n|\mathbf{k} \sigma><\mathbf{k} \sigma| m>
$$

where the admittance of the plane wave $\mathbf{k} \sigma, Y_{\mathbf{k} \sigma}$, is $g / k_{z}(\mathbf{k})$ for a p-polarized wave and $k_{z}(\mathbf{k}) / g$ for a spolarized one, with $g=2 \pi / \lambda$. An important property that can be extracted from Eq. (3) is that $G_{m n ; \mathbf{k}}$ diverges whenever a p-polarized diffraction wave goes glancing $\left(k_{z}=0\right)$.

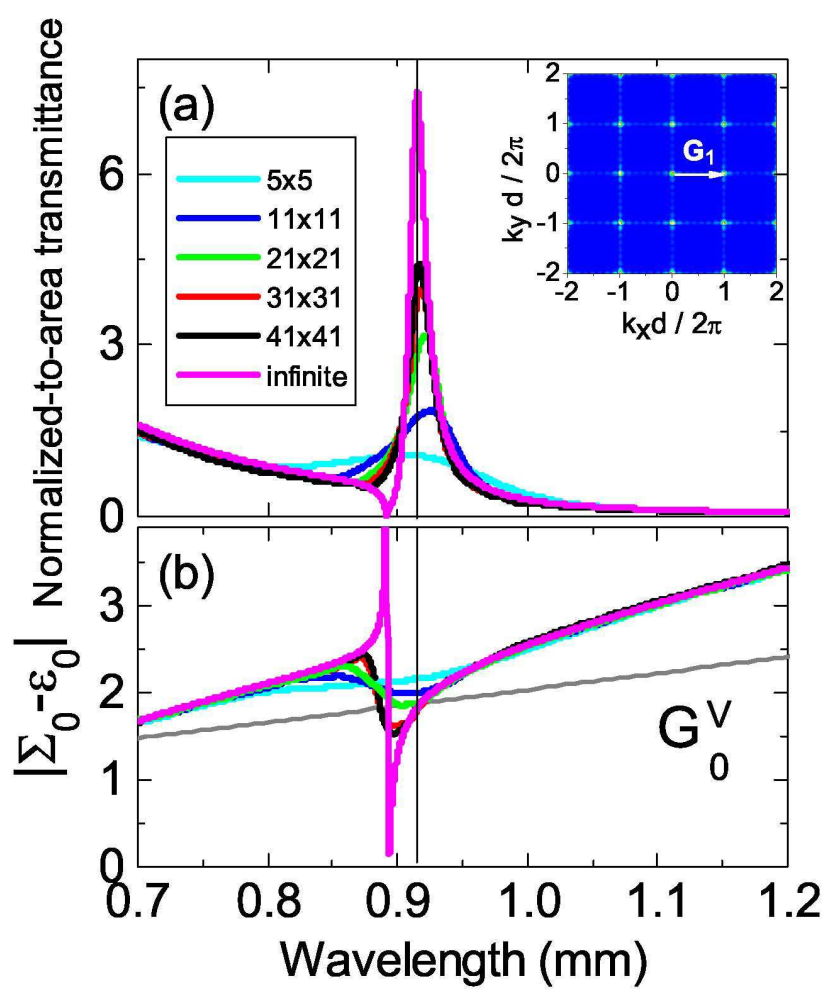

FIG. 2: (color-on-line) (a) Normalized-to-area transmittance versus wavelength for an infinite periodic array (magenta line) and several finite square arrays. The geometrical parameters are: $a=0.2 \mathrm{~mm}, h=0.075 \mathrm{~mm}$ and $P=0.89 \mathrm{~mm}$. Inset shows the structure factor for the $41 \times 41$ case. (b) $\left|\Sigma_{0}-\epsilon_{0}\right|$ and $G_{0}^{V}$ versus wavelength for the cases depicted in (a).

In order to illustrate the mathematics of the formation of surface EM modes, let us consider the simpler system of an infinite periodic array of holes. Additionally, 
we consider normal incidence and assume that only one waveguide mode couples directly to external radiation (the least evanescent mode, labelled as $n=0$ ). By taking advantage of Bloch's theorem $\left[E_{0}\left(\mathbf{k}+\mathbf{G}_{i}\right)=E_{0}(\mathbf{k})\right.$ and $S(\mathbf{k})=\sum_{i} \delta\left(\mathbf{k}-\mathbf{G}_{i}\right)$, being $\mathbf{G}_{i}$ a reciprocal lattice vector], Eqs. (1) for $\mathbf{k}=\mathbf{k}_{0}=\mathbf{0}$ transform into two simple equations for $E_{0}(\mathbf{0})$ and $E_{0}^{\prime}(\mathbf{0})$ :

$$
\begin{aligned}
& \left(\Sigma_{0}-\epsilon_{0}\right) E_{0}(\mathbf{0})-G_{0}^{V} E_{0}^{\prime}(\mathbf{0})=I_{0} \\
& \left(\Sigma_{0}-\epsilon_{0}\right) E_{0}^{\prime}(\mathbf{0})-G_{0}^{V} E_{0}(\mathbf{0})=0
\end{aligned}
$$

where $\Sigma_{0}=\sum_{\mathbf{G}_{i}} G_{00 ; \mathbf{G}_{i}}$. In Figure 2, $T$ (panel a) and $\left|\Sigma_{0}-\epsilon_{0}\right|$ (panel b) versus wavelength are depicted for an infinite periodic array (magenta line). The geometrical parameters of this array are the same as the periodic one analyzed in Fig.1. As $\Sigma_{0}$ for $\mathbf{G}= \pm \mathbf{G}_{1}$ diverges at $\lambda=P=0.89 \mathrm{~mm}$, both $E_{0}(\mathbf{0})$ and $E_{0}^{\prime}(\mathbf{0})$ are zero leading to zero transmission. This is the socalled Wood's anomaly [1] or anti-resonance as quoted in Ref.[14]. The crucial point to realize is that, due to its rapid variation close to the divergence, at a wavelength slightly larger than the one corresponding to glancing angle, $\left|\Sigma_{0}-\epsilon_{0}\right|=G_{0}^{V}$. This leads to a resonant enhancement of the electric field amplitudes at the interfaces of the system [see Eqs.(4)], which can be assigned to the excitation of a leaky surface EM mode [17]. Consequently, $T$ presents a maximum at the corresponding wavelength (see Fig.2). Importantly, this resonance appears through the coupling to p-polarized modes, closely resembling the EM fields of surface plasmons in a real metal. Due to that, these modes are usually called spoof surface plasmons emerging when the surface of a perfect conductor is periodically corrugated [18].

The arguments presented above can be extended to the case of finite arrays. Now Bloch's theorem cannot be applied and the system of Eqs.(1) must be solved for a continuum of states q. However, we have checked that for a finite array with a large number of holes $\mathbf{q}=\mathbf{k}_{0}=\mathbf{0}$ is the dominant transmission channel and the equations for $E_{0}(\mathbf{0})$ and $E_{0}^{\prime}(\mathbf{0})$ could be written like Eqs.(4) with $\Sigma_{0}$ numerically calculated from the knowledge of $E_{0}(\mathbf{k})$ and $E_{0}^{\prime}(\mathbf{k})$ (see Eq.(2)). The results of this approach are shown in Figure 2 for the case of square periodic arrays of holes (going from $5 \times 5$ to $41 \times 41$ ). In this case $\Sigma_{0}$ presents no divergences but resonant features appearing close to $\lambda=P$. The first consequence is that Wood's anomalies do not reach zero-value in finite arrays. As for the infinite case, the cut between $\left|\Sigma_{0}-\epsilon_{0}\right|$ and $G_{0}^{V}$ marks the location of the transmission peak for large arrays $(41 \times 41$ and $31 \times 31$ ). For smaller arrays, there is no cut and the transmission peak appears at the wavelength in which the distance between $\left|\Sigma_{0}-\epsilon_{0}\right|$ and $G_{0}^{V}$ is minimal.

Once described the periodic case, it is straightforward to analyze the case of a quasiperiodic array of holes. In panel (b) of Figure 3, the evolution of $\left|\Sigma_{0}-\epsilon_{0}\right|$ versus wavelength is studied for Penrose lattices with increasing

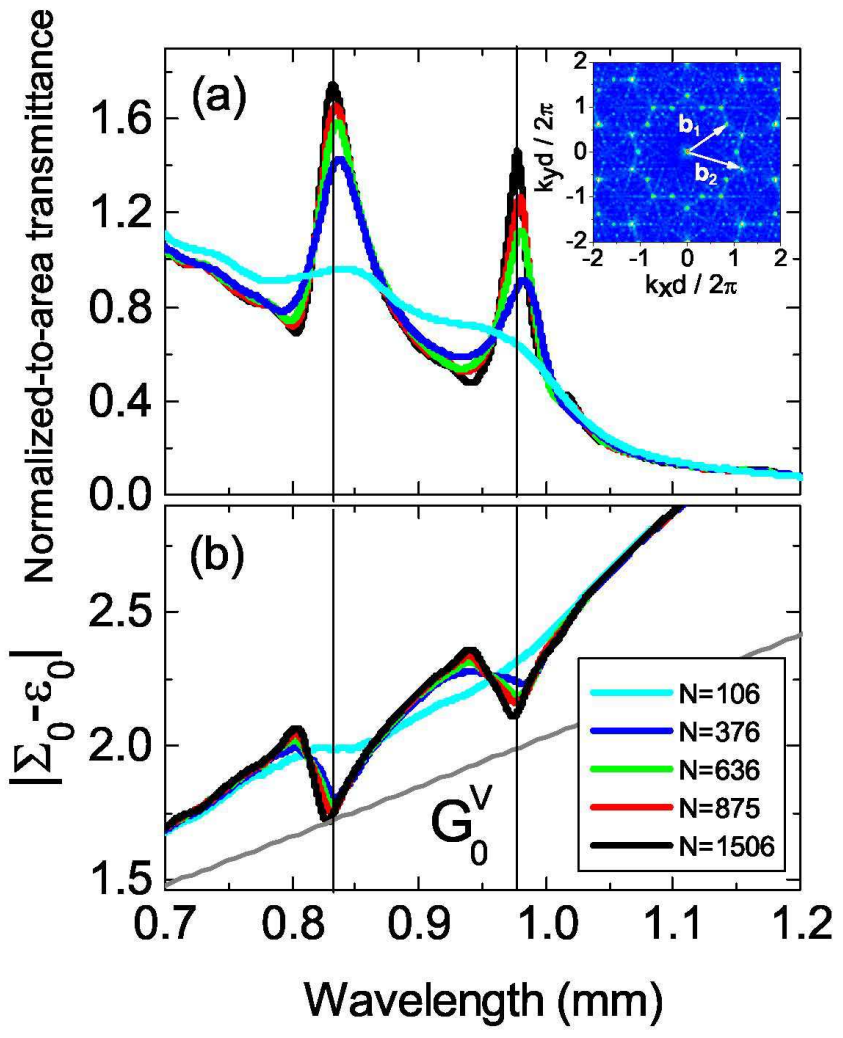

FIG. 3: (color-on-line)(a) Normalized-to-area transmittance versus wavelength for several quasiperiodic arrays with different number of holes, $N$. The geometrical parameters are: $a=0.2 \mathrm{~mm}, h=0.075 \mathrm{~mm}$ and $d=1 \mathrm{~mm}$. Inset shows the structure factor for the $N=1506$ case. (b) Both $\left|\Sigma_{0}-\epsilon_{0}\right|$ and $G_{0}^{V}$ versus wavelength for the cases depicted in (a).

number of holes (ranging from $N=106$ to $N=1506$, the case analyzed in Fig.1). $\left|\Sigma_{0}-\epsilon_{0}\right|$ present maxima at wavelengths corresponding to the glancing condition for the two main wavevectors of the structure factor (see inset of Fig.3a): $\vec{b}_{1}\left(\lambda_{1}=0.8 \mathrm{~mm}\right)$ and $\vec{b}_{2}\left(\lambda_{2}=0.94 \mathrm{~mm}\right)$. Consequently, $T$ shows two minima at these two wavelengths. At slightly larger wavelengths, the distance between $\left|\Sigma_{0}-\epsilon_{0}\right|$ and $G_{0}^{V}$ is minimal and, correspondingly, two transmission peaks appear in the spectrum. Therefore, these resonant transmission peaks stem from the excitation of surface EM modes at the metallic surfaces, much in the same way as in periodic arrays. Notice that, however, in the quasiperiodic case, there is no minimum wavevector for diffraction (i.e. the structure factor is non-zero for wavevectors with moduli smaller than $\left|\vec{b}_{1}\right|$, see inset of Fig.3a). This results in diffraction onto additional propagating modes in vacuum (other than the zero-order mode), which leads to both smaller resonant peaks and less pronounced Wood's anomalies than those emerging in the periodic case.

It is worth analyzing how is the spatial distribution of light emerging from the quasiperiodic array. Figure 4 renders the transmission-per-hole in a Penrose lattice 

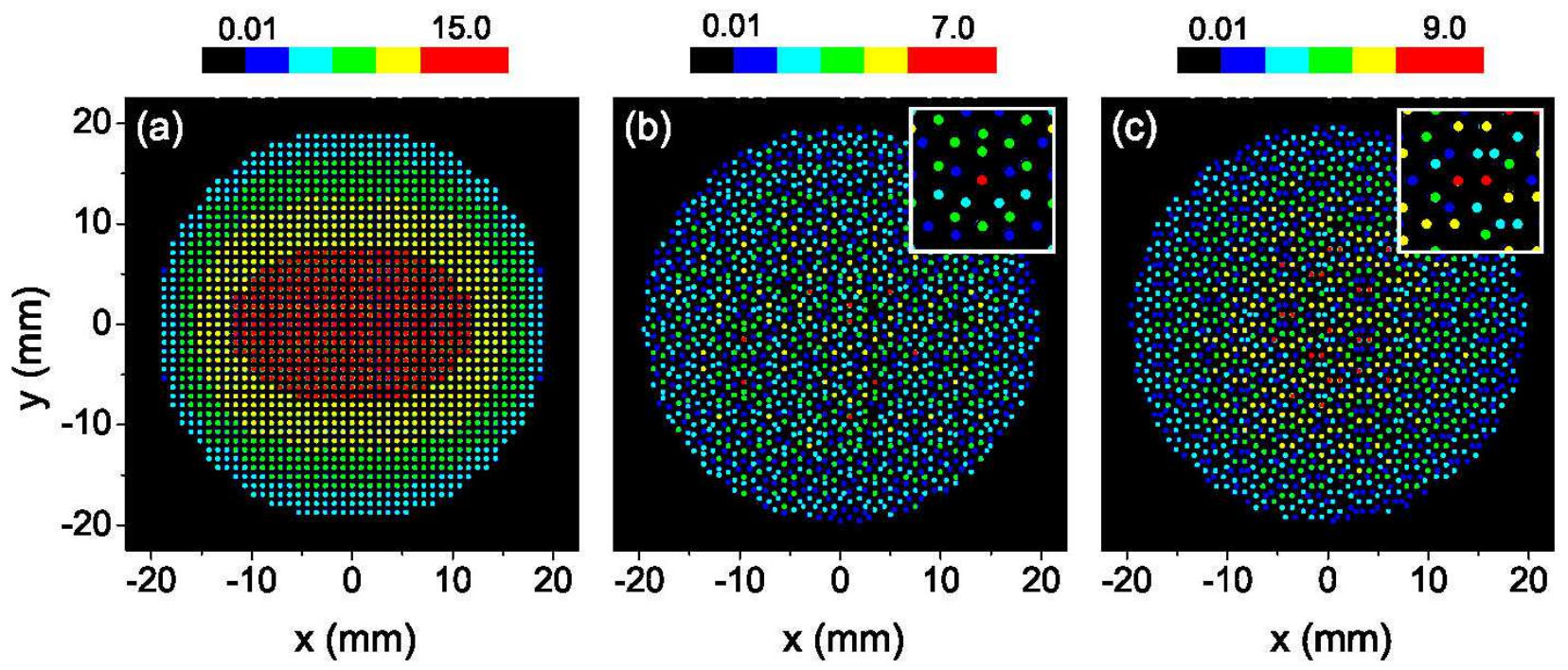

FIG. 4: (color) Transmission per hole (normalized to the single hole transmission) displayed in a color scale for (a) ordered case evaluated at $\lambda=0.92 \mathrm{~mm}$, (b) Penrose lattice at $\lambda=0.83 \mathrm{~mm}$ and (c) Penrose lattice at $\lambda=0.98 \mathrm{~mm}$. The geometrical parameters are the same as in Figure 1.

of $N=1506$ holes at the two resonant wavelengths $(\lambda=0.83 \mathrm{~mm}$ and $\lambda=0.98 \mathrm{~mm}$ in panels $(\mathrm{b})$ and $(\mathrm{c})$ of Fig.4, respectively). For comparison, panel (a) of Fig. 4 shows the corresponding distribution for the ordered array at the resonant wavelength $0.92 \mathrm{~mm}$. In all three cases, incident $\mathbf{E}$-field is pointing along the $x$-direction. In the ordered case, due to finite size effects, the maximum transmission is located at the center of the structure [19]. In quasiperiodic arrangements, the transmissionper-hole distribution presents a completely different pattern: it is far from being uniform, showing the appearance of some holes with high transmission (hot-spots), which are highlighted in the insets of panels (b) and (c). Interestingly, in the Penrose lattice, for a given resonant wavelength, hot spots show similar local environment. However, the existence of hot spots does not imply that EOT in quasiperiodic systems is dominated by very localized resonant configurations of holes. Calculations (not shown here) on finite clusters of holes centered at the hot spots show an increase of transmittance as a function of number of neighbors included in the cluster. This point is reinforced by the fact that the resonant peaks observed in the transmission spectra of finite Penrose lattices do not saturate for small $N$ values (see inset of Fig. 1d). Both these results are consistent with the interpretation based on extended leaky surface EM modes described above.

In conclusion, by developing a k-space theoretical formalism, we have been able to demonstrate that the resonant features observed in the transmission spectra of $2 \mathrm{D}$ Penrose lattices of holes can be explained in terms of the formation of surface EM modes at the interfaces of the metal film. Furthermore, we have linked the formation of these modes to the structure factor of the hole arrays, enabling the understanding of the appearance of extraor- dinary optical transmission in more general conditions.

Financial support by the Spanish MECD under grant BES-2003-0374 and contract MAT2005-06608-C02 is gratefully acknowledged.

[1] T.W. Ebbesen et al., Nature 391, 667 (1998).

[2] L. Martin-Moreno et al., Phys. Rev. Lett. 86, 1114 (2001).

[3] J. Gomez Rivas et al., Phys. Rev. B 68, 201306(R) (2003).

[4] R. Gordon et al., Phys. Rev. Lett. 92, 037401 (2004).

[5] W.L. Barnes et al., Phys. Rev. Lett. 92, 107401 (2004).

[6] K.J. Klein Koerkamp et al., Phys. Rev. Lett. 92, 183901 (2004).

[7] J. Bravo-Abad, F.J. García-Vidal, and L. MartínMoreno, Phys. Rev. Lett. 93, 227401 (2004).

[8] J.T. Shen, P.B. Catrysse, and S. Fan, Phys. Rev. Lett. 94, 197401 (2005).

[9] P. Lalanne, J.C. Rodier, and J.P. Hugonin, J. Opt. Pure Appl. Opt. 7, 422 (2005).

[10] A.P. Hibbins et al. Phys. Rev. Lett. 96, 073904 (2006).

[11] M. Sun et al., Chin. Phys. Lett. 23, 486 (2006).

[12] A.S. Schwanecke et al., OSA Nanophotonics Topical Meeting, April 2006 (post-deadline).

[13] F. Przybilla, C. Genet, and T.W. Ebbesen, Appl. Phys. Lett. 89, 121115 (2006).

[14] T. Matsui et al., Nature 446, 517 (2007).

[15] D. Levine and P. J. Steinhardt, Phys. Rev. B 34, 596 (1986).

[16] D. A. Rabson, N. D. Mermin, D. S. Rokshar and D. C. Wright, Rev. Mod. Phys 63, 699 (1991).

[17] A.D. Boardman, Electromagnetic Surface Modes (Wiley, New York, 1982).

[18] J. B. Pendry, L. Martín-Moreno, and F.J. García-Vidal, Science 305, 847 (2004).

[19] J. Bravo-Abad et al., Nature Physics 2, 120 (2006). 